\title{
Approximate Best Linear Unbiased Channel Estimation for Frequency Selective Channels with Long Delay Spreads: Robustness to Timing and Carrier Offsets
}

\author{
Serdar Özen*, S. M. Nerayanuru ${ }^{\dagger}$, Christopher Pladdy ${ }^{\dagger}$, and Mark J. Fimoff ${ }^{\dagger}$ \\ * Department of Electrical \& Electronics Engineering, \\ İzmir Institute of Technology \\ Urla, İzmir - Turkey \\ Phone: +90 2327506580 \\ serdarozen@iyte.edu.tr \\ ${ }^{\dagger}$ LG Electronics Zenith Research Center \\ Lincolnshire, IL 60069 - USA \\ Phone: (847) 9418595 \\ \{snerayanuru, christopher.pladdy, mark.fimoff \}@zenith.com
}

\begin{abstract}
We provide an iterative and a non-iterative channel impulse response (CIR) estimation algorithm for communication systems which utilize a periodically transmitted training sequence within a continuous stream of information symbols. The iterative procedure calculates the (semi-blind) Best Linear Unbiased Estimate (BLUE) of the CIR. The non-iterative version is an approximation to the BLUE CIR estimate, denoted by aBLUE, achieving almost similar performance, with much lower complexity. Indeed we show that, with reasonable assumptions, a-BLUE channel estimate can be obtained by using a stored copy of a pre-computed matrix in the receiver which enables the use of the initial CIR estimate by the subsequent equalizer tap weight calculator. Simulation results are provided to demonstrate the performance of the novel algorithms for 8-VSB ATSC Digital TV system. We also provide a simulation study of the robustness of the a-BLUE algorithm to timing and carrier phase offsets.
\end{abstract}

KEY WORDS: channel estimation, least squares, best linear unbiased estimator, signal processing for communications applications, carrier and timing offsets

\section{INTRODUCTION}

For the communications systems utilizing periodically transmitted training sequence, least-squares (LS) based channel estimation or the correlation based channel estimation algorithms have been the most widely used two alternatives [1]. Both methods use a stored copy of the known transmitted training sequence at the receiver. The properties and the length of the training sequence are generally different depending on the particular communication system's standard specifications. However most channel estimation schemes ignore the baseline noise term which occurs due to the correlation of the stored copy of the training sequence with the unknown symbols adjacent to transmitted training sequence, as well as the additive channel noise [1], [9]. In the sequel, we provide (semi-blind) Best Linear Unbiased Estimate (BLUE) and approximate
BLUE (a-BLUE) channel estimators for communication systems using a periodically transmitted training sequence. Our novel CIR estimation algorithms can be considered as semiblind techniques since these methods take advantage of the statistics of the data [3]. Although the examples following the derivations of the BLUE and the a-BLUE channel estimators will be drawn from the ATSC digital TV 8-VSB system [2], to the best of our knowledge it could be applied with minor modifications to any digital communication system with linear modulation which employs a periodically transmitted training sequence. The novel algorithm presented in the sequel is targeted for the systems that are desired to work with channels having long delay spreads $L_{d}$; in particular we consider the case where $(N T+1) / 2<L_{d}<N T$, where $N T$ is the duration of the available training sequence. For instance the 8-VSB digital TV system has 728 training symbols, whereas the delay spreads of the terrestrial channels have been observed to be at least 400-500 symbols long [4], [5]. The a-BLUE algorithm can be used as an initializer to the BLUE iterations[7], or as a stand-alone alternative[6] approach that produces results of nearly the same quality as the results produced by the BLUE algorithm while at the same time requiring much less computational complexity (i.e., requiring about the same number of multiplications necessary to implement ordinary least squares) and having storage requirements similar to that of ordinary least squares.

\section{A. Overview of Generalized Least Squares}

Consider the linear model

$$
\boldsymbol{y}=\boldsymbol{A x}+\boldsymbol{\nu}
$$

where $\boldsymbol{y}$ is the observation (or response) vector, $\boldsymbol{A}$ is the regression (or design) matrix, $\boldsymbol{x}$ is the vector of unknown 
parameters to be estimated, and $\boldsymbol{\nu}$ is the observation noise (or measurement error) vector. Assuming that it is known that the random noise vector $\nu$ is zero mean, and is correlated, that is $\operatorname{Cov}\{\boldsymbol{\nu}\}=\boldsymbol{K}_{\nu} \equiv \frac{1}{2} E\left\{\boldsymbol{\nu} \boldsymbol{\nu}^{H}\right\} \neq \sigma_{\boldsymbol{\nu}}^{2} \boldsymbol{I}$, we define the (generalized) objective function for the model of (1) by

$$
J_{G L S}(\boldsymbol{x})=(\boldsymbol{y}-\boldsymbol{A} \boldsymbol{x})^{H} \boldsymbol{K}_{\nu}^{-1}(\boldsymbol{y}-\boldsymbol{A} \boldsymbol{x}) .
$$

The least squares estimate that minimizes Equation (2) is

$$
\hat{\boldsymbol{x}}_{g l s}=\left(\boldsymbol{A}^{H} \boldsymbol{K}_{\nu}^{-1} \boldsymbol{A}\right)^{-1} \boldsymbol{A}^{H} \boldsymbol{K}_{\nu}^{-1} \boldsymbol{y}
$$

The estimator of (3) is called the best linear unbiased estimate (BLUE) [8] among all linear unbiased estimators if the noise covariance matrix is known to be $\operatorname{Cov}\{\boldsymbol{\nu}\}=\boldsymbol{K}_{\nu}$. The estimator of (3) is called the minimum variance unbiased estimator (MVUE) among all unbiased estimators (not only linear) if the noise is known to be Gaussian with zero mean and with covariance matrix $\boldsymbol{K}_{\nu}$, that is $\hat{\boldsymbol{x}}_{\text {ols }}$ is called MVUE if it is known that $\boldsymbol{\nu} \sim \mathcal{N}\left(\mathbf{0}, \boldsymbol{K}_{\nu}\right)$.

\section{OvERVIEW OF DATA Transmission Model}

The baseband symbol rate sampled receiver pulse-matched filter output is given by

$$
\begin{aligned}
y[n] & \left.\equiv y(t)\right|_{t=n T}=\sum_{k} I_{k} h[n-k]+\nu[n] \\
& =\sum_{k} I_{k} h[n-k]+\sum_{k} \eta[k] q^{*}[-n+k],
\end{aligned}
$$

where $I_{k}=\left\{\begin{array}{ll}a_{k}, & 0 \leq k \leq N-1 \\ d_{k}, & N \leq n \leq N^{\prime}-1,\end{array}\right\} \in \mathcal{A} \equiv\left\{\alpha_{1}, \cdots, \alpha_{M}\right\}$ is the $M$-ary complex valued transmitted sequence, $\mathcal{A} \subset \mathbb{C}^{1}$, and $\left\{a_{k}\right\} \in \mathbb{C}^{1}$ denote the first $N$ symbols within a frame of length $N^{\prime}$ to indicate that they are the known training symbols, and the remaining $N^{\prime}-N$ symbols $\left\{d_{k}\right\} \in \mathbb{C}^{1}$ are the information symbols; $\nu[n]=\eta[n] * q^{*}[-n]$ denotes the complex (colored) noise process after the (pulse) matched filter, with $\eta[n]$ being a zero-mean white Gaussian noise process with variance $\sigma_{\eta}^{2}$ per real and imaginary part;

$$
h(t)=q(t) * c(t) * q^{*}(t)=\sum_{k=-K}^{L} c_{k} p\left(t-\tau_{k}\right)
$$

is the complex valued impulse response of the composite channel, including pulse shaping transmit filter $q(t)$, the physical channel impulse response $c(t)$, and the receive filter $q^{*}(-t) ; p(t)=q(t) * q^{*}(-t)$ is the convolution of the transmit and receive filters where $q(t)$ has a finite support of $\left[-T_{q} / 2, T_{q} / 2\right]$, and the span of the transmit and receive filters, $T_{q}$, is an even multiple of the symbol period, $T$; that is $T_{q}=N_{q} T, N_{q}=2 L_{q} \in \mathbb{Z}^{+} .\left\{c_{k}\right\} \subset \mathbb{C}^{1}$ denote complex valued physical channel gains, and $\left\{\tau_{k}\right\}$ denote the multipath delays, or the Time-Of-Arrivals (TOA). It is assumed that the time-variations of the channel is slow enough that $c(t)$ can be assumed to be a static inter-symbol interference (ISI) channel, at least throughout the training period. Without loss of generality, the symbol rate sampled composite CIR, $h[n]$, can be written as a finite dimensional vector
$\boldsymbol{h}=\left[h\left[-N_{a}\right], \cdots, h[-1], h[0], h[1], \cdots, h\left[N_{c}\right]\right]^{T}$ where $N_{a}$ and $N_{c}$ denote the number of anti-causal and causal taps of the channel, respectively, and $L_{d}=\left(N_{a}+N_{c}+1\right) T$ is the delay spread of the channel (including the pulse tails). The pulse matched filter output which includes all the contributions from the known training symbols (which includes the adjacent random data as well) can be written as ${ }^{1}$

$$
\begin{aligned}
\boldsymbol{y}_{\left[-N_{a}: N+N_{c}-1\right]} & =(\boldsymbol{A}+\boldsymbol{D}) \boldsymbol{h}+\boldsymbol{\nu}_{\left[-N_{a}: N+N_{c}-1\right]} \\
& =\boldsymbol{A} \boldsymbol{h}+\boldsymbol{D} \boldsymbol{h}+\boldsymbol{Q} \boldsymbol{\eta}_{\left[-N_{a}-L_{q}: N+N_{c}-1+L_{q}(\text { (). })\right.} \\
& =\boldsymbol{A} \boldsymbol{h}+\boldsymbol{H} \boldsymbol{d}+\boldsymbol{Q} \boldsymbol{\eta}_{\left[-N_{a}-L_{q}: N+N_{c}-1+L_{q}(7)\right.}
\end{aligned}
$$

where $\boldsymbol{A}=\mathcal{T}\left\{[a_{0}, \cdots, a_{N-1}, \underbrace{0, \cdots, 0}_{N_{a}+N_{c}}]^{T},[a_{0}, \underbrace{0, \cdots, 0}_{N_{a}+N_{c}}]\right\}$, is a Toeplitz matrix ${ }^{2}$ of dimension $(N+$ $\left.N_{a}+N_{c}\right) \times\left(N_{a}+N_{c}+1\right)$, and $\boldsymbol{D}=$ $\mathcal{T}\left\{[\underbrace{0, \cdots, 0}_{N}, d_{N}, \cdots, d_{N_{c}+N_{a}+N-1}]^{T},\left[0, d_{-1}, \cdots, d_{-N_{c}-N_{a}}\right]\right\}$,

is a Toeplitz matrix which includes the adjacent unknown symbols, prior to and after the training sequence. The data sequence $\left[d_{-1}, \cdots, d_{-N_{c}-N_{a}}\right]$ is the unknown information symbols transmitted at the end of the frame prior to the current frame being transmitted. $Q$ is of dimension $\left(N+N_{a}+N_{c}\right) \times\left(N+N_{a}+N_{c}+N_{q}\right)$ and is given by $\boldsymbol{Q}=\left[\begin{array}{cccc}\boldsymbol{q}^{T} & 0 & \cdots & 0 \\ 0 & \boldsymbol{q}^{T} & \cdots & 0 \\ \vdots & \vdots & \ddots & \vdots \\ 0 & 0 & \cdots & \boldsymbol{q}^{T}\end{array}\right]$ $\boldsymbol{q}=\left[q\left[+L_{q}\right], \cdots, q[0], \cdots, q\left[-L_{q}\right]\right]^{T}$, and

$$
\begin{aligned}
\boldsymbol{H}= & \mathcal{H} \boldsymbol{S}^{T}, \\
\overline{\boldsymbol{h}}= & {\left[h\left[N_{c}\right], \cdots, h[1], h[0], h[-1], \cdots, h\left[-N_{a}\right]\right]^{T}=\boldsymbol{J}(\boldsymbol{\theta},) } \\
\boldsymbol{J}= & {\left[\begin{array}{cccc}
0 & \cdots & 0 & 1 \\
0 & \cdots & 1 & 0 \\
\vdots & \vdots & & \vdots \\
1 & 0 & \cdots & 0
\end{array}\right]_{\left(N_{a}+N_{c}+1\right) \times\left(N_{a}+N_{c}+1\right)}(10) } \\
\mathcal{H}= & {\left[\begin{array}{cccc}
\overline{\boldsymbol{h}}^{T} & 0 & \cdots & 0 \\
0 & \overline{\boldsymbol{h}}^{T} & \cdots & 0 \\
\vdots & \vdots & \ddots & \vdots \\
0 & 0 & \cdots & \overline{\boldsymbol{h}}^{T}
\end{array}\right]_{\left(N+N_{c}+N_{a}\right) \times\left(N+2\left(N_{a}+N_{c}\right)\right)},(11) }
\end{aligned}
$$

and

\footnotetext{
${ }^{1}$ The notation of $\boldsymbol{y}_{\left[n_{1}: n_{2}\right]}$ with $n_{2} \geq n_{1}$ indicates the column vector

$$
\boldsymbol{y}_{\left[n_{1}: n_{2}\right]}=\left[y\left[n_{1}\right], y\left[n_{1}+1\right], \cdots, y\left[n_{2}\right]\right]^{T} .
$$
}

Same notation will also be applied to the noise variables $\nu[n], \eta[n]$, where $\nu[n]$ is the complex AWGN at the input to receive filter, $\eta[n]$ is the colored noise at the output of the receive filter.

${ }^{2}$ The notation of $\mathcal{T}\left\{\boldsymbol{a}, \boldsymbol{b}^{T}\right\}$ stands for a Toeplitz matrix of dimension $M \times N$ with first column $\boldsymbol{a}=\left[a_{0}, \ldots, a_{M-1}\right]^{T}$ and first row $\boldsymbol{b}=$ $\left[b_{0}, \ldots, b_{N-1}\right]^{T}$, with $a_{0}=b_{0}$. 
and $\boldsymbol{d}=\boldsymbol{S} \tilde{\boldsymbol{d}}$, or equivalently $\tilde{\boldsymbol{d}}=\boldsymbol{S}^{T} \boldsymbol{d}$, where

$$
\begin{aligned}
& \tilde{\boldsymbol{d}}=\left[d_{-N_{c}-N_{a}}, \cdots, d_{-1}, \mathbf{0}_{1 \times N}, d_{N}, \cdots, d_{N+N_{c}+N_{a}-1}\right]^{T} \\
& \boldsymbol{d}=\left[d_{-N-N_{a}}, \cdots, d_{-1}, d_{N}, \cdots, d_{N+N_{c}+N_{a}-1}\right]^{T} \\
& \boldsymbol{S}=\left[\begin{array}{ccc}
\boldsymbol{I}_{N_{a}+N_{c}} & \boldsymbol{0}_{\left(N_{a}+N_{c}\right) \times N} & \boldsymbol{0}_{\left(N_{a}+N_{c}\right)} \\
\mathbf{0}_{\left(N_{a}+N_{c}\right)} & \mathbf{0}_{\left(N_{a}+N_{c}\right) \times N} & \boldsymbol{I}_{N_{a}+N_{c}}
\end{array}\right] .
\end{aligned}
$$

where $\bar{h}$ is the time reversed version of $\boldsymbol{h}$ (re-ordering is accomplished by the permutation matrix $\boldsymbol{J}$ ), and $\boldsymbol{H}$ is of dimension $\left(N+N_{a}+N_{c}\right) \times\left(2\left(N_{c}+N_{a}\right)\right)$ with a "hole" inside which is created by the selection matrix $\boldsymbol{S}$, where $\boldsymbol{S}$ is $\left(2\left(N_{c}+N_{a}\right)\right) \times\left(N+2\left(N_{a}+N_{c}\right)\right)$ dimensional selection matrix which retains the random data, eliminates the $N$ zeros in the middle of the vector $\tilde{\boldsymbol{d}}$.

\section{OVERVIEW OF THE BLUE CIR ESTIMATOR}

For comparison purposes we first provide the well known correlation and ordinary least squares based estimators, where correlations based estimation is denoted $\hat{\boldsymbol{h}}_{u}$ (the subscript $u$ stands for the uncleaned CIR estimate) and is given by

$$
\hat{\boldsymbol{h}}_{u}=\frac{1}{r_{a}[0]} \boldsymbol{A}^{H} \boldsymbol{y}_{\left[-N_{a}: N+N_{c}-1\right]}
$$

with $r_{a}[0]=\sum_{k=0}^{N-1}\left\|a_{k}\right\|^{2}$, and the ordinary least squares CIR estimate is denoted by $\hat{\boldsymbol{h}}_{c}$ (the subscript ${ }_{c}$ stands for the cleaned CIR estimate) and is given by

$$
\hat{\boldsymbol{h}}_{c}=\left(\boldsymbol{A}^{H} \boldsymbol{A}\right)^{-1} \boldsymbol{A}^{H} \boldsymbol{y}_{\left[-N_{a}: N+N_{c}-1\right]},
$$

where "cleaning" is accomplished by removing the known sidelobes of the aperiodic correlation operation which is accomplished in (16).

We can denote the two terms on the right side of Equation (7) by $\boldsymbol{v}=\boldsymbol{H} \boldsymbol{d}+\boldsymbol{Q} \boldsymbol{\eta}_{\left[-N_{a}-L_{q}: N+N_{c}-1+L_{q}\right]}$. Hence we rewrite (7) as

$$
\boldsymbol{y}_{\left[-N_{a}: N+N_{c}-1\right]}=\boldsymbol{A} \boldsymbol{h}+\boldsymbol{v} .
$$

By noting the statistical independence of the random vectors $\boldsymbol{d}$ and $\boldsymbol{\eta}$, and also noting that both vectors are zero mean, the covariance matrix, $\boldsymbol{K}_{\boldsymbol{v}}$ of $\boldsymbol{v}$ is given by

$$
\operatorname{Cov}\{\boldsymbol{v}\}=\boldsymbol{K}_{\boldsymbol{v}} \equiv \frac{1}{2} E\left\{\boldsymbol{v} \boldsymbol{v}^{H}\right\}=\frac{\mathcal{E}_{d}}{2} \boldsymbol{H} \boldsymbol{H}^{H}+\sigma_{\eta}^{2} \boldsymbol{Q} \boldsymbol{Q}^{H},
$$

where $\mathcal{E}_{d}$ is the energy of the transmitted information symbols, and equals to 21 if the symbols $\left\{d_{k}\right\}$ are chosen from the set $\{ \pm 1, \pm 3, \pm 5, \pm 7\}$. For the model of (18) the generalized least squares objective function to be minimized is

$$
J_{G L S}(\boldsymbol{h})=\left(\boldsymbol{y}_{\left[-N_{a}: N+N_{c}-1\right]}-\boldsymbol{A} \boldsymbol{h}\right)^{H} \boldsymbol{K}_{\boldsymbol{v}}^{-1}\left(\boldsymbol{y}_{\left[-N_{a}: N+N_{c}-1\right]}-\boldsymbol{A} \boldsymbol{h}\right) \text {. }
$$

Then the generalized least-squares solution to the model of Equation (18) which minimizes the objective function of $J_{G L S}(\boldsymbol{y})$ is given by

$$
\hat{\boldsymbol{h}}_{K}=\left(\boldsymbol{A}^{H} \boldsymbol{K}_{\boldsymbol{v}}^{-1} \boldsymbol{A}\right)^{-1} \boldsymbol{A}^{H} \boldsymbol{K}_{\boldsymbol{v}}^{-1} \boldsymbol{y}_{\left[-N_{a}: N+N_{c}-1\right]} .
$$

The problem with Equation (21) is that the channel estimate $\hat{\boldsymbol{h}}_{K}$ is based on the covariance matrix $\boldsymbol{K}_{\boldsymbol{v}}$, which is a function of the true channel impulse response vector $\boldsymbol{h}$ as well as the channel noise variance $\sigma_{\eta}^{2}$. In actual applications the BLUE channel estimate of Equation (21) can not be exactly obtained. Hence we need an iterative technique to calculate generalized least squares estimate of (21) where every iteration produces an updated estimate of the covariance matrix as well as the noise variance. Without going into the details, a simplified version of the iterations, which yield a closer approximation to the exact BLUE CIR estimate after each step, is provided in Algorithm 1. In the intermediate steps noise variance is estimated by $\sigma_{\eta}^{2}=\frac{1}{2 \mathcal{E}_{q}\left(N-N_{a}-N_{c}\right)} \| \hat{\boldsymbol{y}}_{\left[N_{c}: N-N_{a}\right]}-$ $\boldsymbol{y}_{\left[N_{c}: N-N_{a}\right]} \|^{2}$, where $\mathcal{E}_{q}=\|\boldsymbol{q}\|^{2}$ and $\hat{\boldsymbol{y}}_{\left[N_{c}: N-N_{a}\right]}=\tilde{\boldsymbol{A}} \hat{\boldsymbol{h}}_{t h}$, $\tilde{\boldsymbol{A}}=\mathcal{T}\left\{\left[a_{N_{c}+N_{a}}, \cdots, a_{N-1}\right]^{T},\left[a_{N_{c}+N_{a}}, \cdots, a_{0}\right]\right\}$. For further details regarding the BLUE algorithm, the readers are referred to [7].

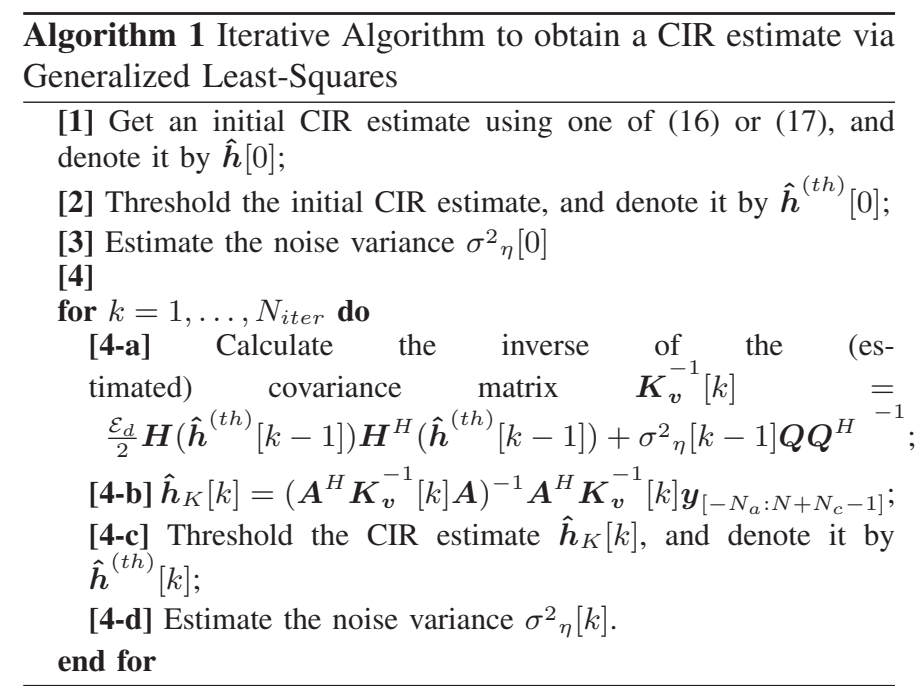

\section{A. Approximate BLUE CIR estimation}

An alternative approach may be used to produce results of nearly the same quality as the results produced by the algorithm described in Algorithm 1 while at the same time requiring much less computational complexity (i.e., requiring about the same number of multiplications necessary to implement Equation (17)) and having storage requirements similar to that of Equation (17). According to this alternative, the initial least squares estimation error can be reduced by seeking an approximation in which it is assumed that the baseband representation of the physical channel $c(t)$ is a distortion-free (no multipath) channel; that is

$$
c(t)=\delta(t)
$$

which implies $h(t)=p(t) * c(t)=p(t)$. Thus we can assume that our finite length channel impulse response vector can be (initially) approximated by

$$
\tilde{\boldsymbol{h}}=[\underbrace{0, \cdots, 0}_{N_{a}-N_{q}}, \underbrace{p\left[-N_{q}\right], \cdots, p[0], \cdots, p\left[N_{q}\right.}_{\text {raised cosine pulse }}], \underbrace{0, \cdots, 0}_{N_{c}-N_{q}}]^{T}
$$


with the assumptions of $N_{a} \geq N_{q}$ and $N_{c} \geq N_{q}$, that is the tail span of the composite pulse shape is well confined to within the assumed delay spread of $\left[-N_{a} T, N_{c} T\right]$. Then the approximation of (23) can be substituted into Equations (1012) to yield an initial (approximate) channel convolution matrix $\tilde{\boldsymbol{H}}$ and is given by $\tilde{\boldsymbol{H}}=\tilde{\mathcal{H}} S^{T}$ where $\tilde{\mathcal{H}}$ is formed as in Equation (12) with $\overline{\tilde{h}}=\boldsymbol{J} \tilde{\boldsymbol{h}}$. We can also assume a reasonable received Signal-to-Noise (SNR) ratio measured at the input to the matched filter which is given by

$$
\mathrm{SNR}=\frac{\mathcal{E}_{d}\left\|\left.(c(t) * q(t))\right|_{t=n T}\right\|^{2}}{\sigma_{\eta}^{2}}=\frac{\mathcal{E}_{d}\|\boldsymbol{q}\|^{2}}{\sigma_{\eta}^{2}} .
$$

For instance we can assume an approximate SNR of $20 \mathrm{~dB}$ yielding an initial noise variance of $\tilde{\sigma}_{\eta}^{2}=\frac{\mathcal{E}_{d}\|\boldsymbol{q}\|^{2}}{100}$. Then combining $\tilde{\boldsymbol{H}}$ and $\tilde{\sigma}_{\eta}^{2}$ we can pre-calculate the initial approximate covariance matrix where the covariance matrix of the approximate channel is given by

$$
\tilde{\boldsymbol{K}}_{\boldsymbol{v}}(\tilde{\boldsymbol{H}})=\frac{1}{2} \mathcal{E}_{d} \tilde{\boldsymbol{H}} \tilde{\boldsymbol{H}}^{H}+\tilde{\sigma}_{\eta}^{2} \boldsymbol{Q} \boldsymbol{Q}^{H},
$$

which further leads to the initial channel estimate of

$$
\hat{\boldsymbol{h}}_{\tilde{K}}=\underbrace{\left(\boldsymbol{A}^{H}\left[\tilde{\boldsymbol{K}}_{\boldsymbol{v}}(\tilde{\boldsymbol{H}})\right]^{-1} \boldsymbol{A}\right)^{-1} \boldsymbol{A}^{H}\left[\tilde{\boldsymbol{K}}_{\boldsymbol{v}}(\tilde{\boldsymbol{H}})\right]^{-1}}_{\text {pre-computed and stored }} \boldsymbol{y}_{\left[-N_{a}: N+N_{c}-1\right]} \text {. }
$$

Equation (26) is the resulting a-BLUE CIR estimate. The key advantage of the a-BLUE is that the matrix

$$
\left(\boldsymbol{A}^{H}\left[\tilde{\boldsymbol{K}}_{\boldsymbol{v}}(\tilde{\boldsymbol{H}})\right]^{-1} \boldsymbol{A}\right)^{-1} \boldsymbol{A}^{H}\left[\tilde{\boldsymbol{K}}_{\boldsymbol{v}}(\tilde{\boldsymbol{H}})\right]^{-1}
$$

is constructed based on the initial assumptions that the receiver is expected to operate, and can be pre-computed and stored in the receiver. By pre-computing and storing the matrix $\left(\boldsymbol{A}^{H}\left[\tilde{\boldsymbol{K}}_{\boldsymbol{v}}(\tilde{\boldsymbol{H}})\right]^{-1} \boldsymbol{A}\right)^{-1} \boldsymbol{A}^{H}\left[\tilde{\boldsymbol{K}}_{\boldsymbol{v}}(\tilde{\boldsymbol{H}})\right]^{-1}$ as in Equation (26) we obtain a CIR estimate with much lower computational complexity than the BLUE algorithm, and with comparable complexity as the standard least squares of Equation (17). We also note that a-BLUE CIR estimate can be used either as a stand-alone CIR estimator, or an initial estimate which can be used by the BLUE algorithm; additionally it can be used as an initial CIR estimate to be used in the calculation of the tap weights of a subsequent equalizer.

\section{Simulations}

We considered an 8-VSB [2] receiver with a single antenna. 8-VSB system has a complex raised cosine pulse shape [2]. The CIR we considered is given in Table I. The phase angles of individual paths for all the channels are taken to be $\arg \left\{c_{k}\right\}=$ $\exp \left(-j 2 \pi f_{c} \tau_{k}\right), \quad k=-1, \cdots, 6$ where $f_{c}=\frac{50}{T_{s y m}}$ and $T_{\text {sym }}=92.9 \mathrm{nsec}$. The simulations were run at $28 \mathrm{~dB}$ Signalto-Noise-Ratio (SNR) measured at the input to the receive pulse matched filter, and it is calculated by

$$
\mathrm{SNR}=\frac{\mathcal{E}_{d}\left\|\{c(t) * q(t)\}_{t=k T}\right\|^{2}}{\sigma_{\eta}^{2}} .
$$

Figure 1 shows the simulation results for the test channel provided in Table I. Part (a) shows the actual CIR; part (b)
TABLE I

Simulated CHANNEL DELAYS IN SYMBOL PERIODS, RELATIVE GAINS. $L=-1, K=6$,

$L_{d} \approx\left(1+333+2 N_{q}\right) T=453 T \approx 44 \mu \mathrm{SEC}, N_{q}=60$.

\begin{tabular}{|c|c|c|}
\hline Channel taps & Delay $\left\{\tau_{k}\right\}$ & Gain $\left\{\left|c_{k}\right|\right\}$ \\
\hline$k=-1$ & -0.957 & 0.7263 \\
\hline Main $k=0$ & 0 & 1 \\
\hline$k=1$ & 3.551 & 0.6457 \\
\hline$k=2$ & 15.250 & 0.9848 \\
\hline$k=3$ & 24.032 & 0.7456 \\
\hline$k=4$ & 29.165 & 0.8616 \\
\hline$k=5$ & 221.2345 & 0.6150 \\
\hline$k=6$ & 332.9810 & 0.4900 \\
\hline
\end{tabular}

shows the correlation based CIR estimate, of Equation (16) $\hat{\boldsymbol{h}}_{u}$; part (c) shows the ordinary LS based CIR estimate of Equation (17) $\hat{\boldsymbol{h}}_{c}$; part (d) shows the a-BLUE CIR estimate of Equation (26) with an assumed SNR of $22 \mathrm{~dB}$; part (e) shows the BLUE based CIR estimate of Algorithm 1, after the first iteration only $\hat{\boldsymbol{h}}_{K}[1]$; part (f) shows the ideal BLUE case for which the true covariance matrix $\boldsymbol{K}_{\boldsymbol{v}}$ is known. Part (f) provides a bound for the rest of the BLUE algorithm. We note superior performance of the BLUE algorithm even after the first iteration, as compared to the correlation based and ordinary least squares based CIR estimation schemes. However iterative BLUE CIR estimation algorithm is computationally very demanding, thus in many applications the approximate BLUE, as shown in part (d), could be sufficiently acceptable as an initial estimate, and a-BLUE outperforms the ordinary LS based CIR estimate of Equation (17). The performance measure is the normalized least-squares error which is defined by $\mathcal{E}_{N L S}=\frac{\|\boldsymbol{h}-\hat{\boldsymbol{h}}\|^{2}}{N_{a}+N_{c}+1}$. Approximate BLUE significantly
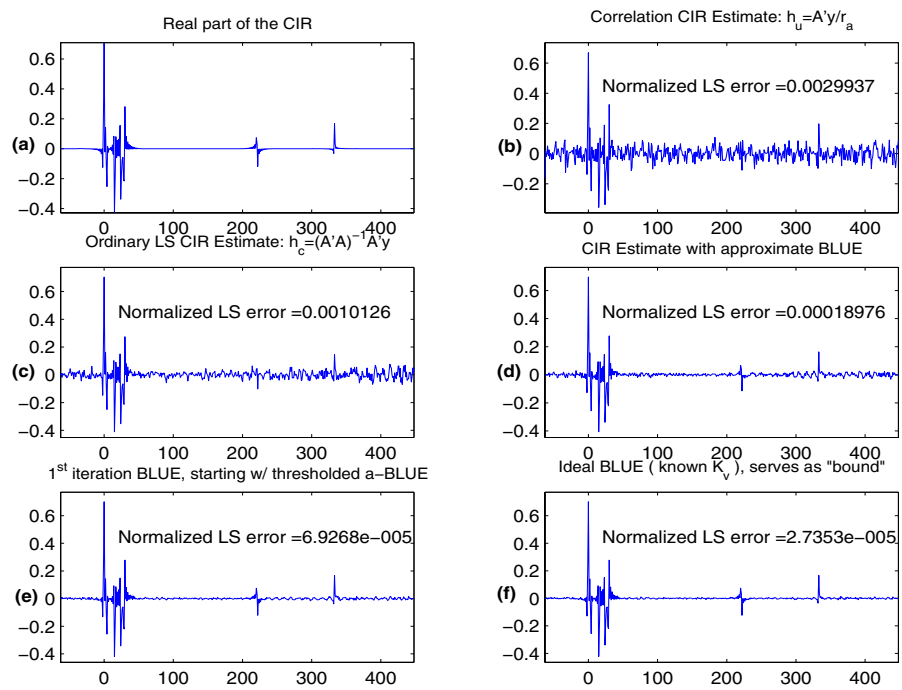

Fig. 1. Part (a) shows the real part of the actual CIR; part (b) shows the correlation based CIR estimate of Equation (16) $\hat{\boldsymbol{h}}_{u}$; part (c) shows the LS based CIR estimate of Equation (17) $\hat{\boldsymbol{h}}_{c}$; part (d) shows the approximate BLUE CIR estimate of Equation (26) with an assumed SNR of 22dB; part (e) show the BLUE based CIR estimate of Algorithm 1, after the first iteration, $\hat{\boldsymbol{h}}_{K}$ [1]; part (f) shows the ideal BLUE case for which the true covariance matrix $\boldsymbol{K}_{\boldsymbol{v}}$ is known, which provides a bound for the rest of the estimators. 
outperforms the ordinary least squares CIR estimation, but it has virtually identical computational complexity and storage requirement.

\section{A. Robustness of the a-BLUE Algorithm to Timing and Carrier Offsets}

The robustness of the a-BLUE CIR estimator to (clock) timing offset and carrier phase offset has also been studied. The assumed channel impulse response shown in Equation (23) consists of perfectly sampled composite pulse shape appearing in the middle of the CIR vector. One may want to investigate the effect of having a receiver timing offset and/or the carrier phase offset on the a-BLUE algorithm.

For timing offset simulations the CIR's are created as

$$
\begin{aligned}
\tilde{\boldsymbol{h}}_{t o}=[\underbrace{0, \cdots, 0}_{N_{a}-N_{q}}, p\left[-N_{q}+\varepsilon_{t o}\right], \cdots, p\left[-1+\varepsilon_{t o}\right], \\
p\left[\varepsilon_{t o}\right], p\left[1+\varepsilon_{t o}\right], \cdots, p\left[N_{q}+\varepsilon_{t o}\right], \underbrace{0, \cdots, 0}_{N_{c}-N_{q}}]^{T}
\end{aligned}
$$

where $\varepsilon_{t o} \in\left(-\frac{T}{2}, \frac{T}{2}\right]$ is the timing offset. Then for the channel of (28) with a fixed $\varepsilon_{t o} \in\left(-\frac{T}{2}, \frac{T}{2}\right]$, we estimated the CIR using Equation (26), and calculated the least squares estimation error $\mathcal{E}_{N L S}$ between the actual CIR and the estimated CIR.

Similarly, for carrier phase offset simulations the CIR's are created as

$$
\begin{aligned}
\tilde{\boldsymbol{h}}_{c o}=\varepsilon_{c o}[\underbrace{0, \cdots, 0}_{N_{a}-N_{q}}, p\left[-N_{q}\right], \cdots, p[-1], \\
p[0], p[1], \cdots, p\left[N_{q}\right], \underbrace{0, \cdots, 0}_{N_{c}-N_{q}}]^{T}
\end{aligned}
$$

where $\varepsilon_{c o}=\exp (-j 2 \pi \theta)$ is the unit complex vector that rotates the original CIR with respect to the offset angle $\theta \in$ $(-\pi, \pi]$. Then for the channel of (29) with a fixed $\varepsilon_{c o}$, we estimated the CIR using Equation (26), and calculated the least squares estimation error $\mathcal{E}_{N L S}$ between the actual CIR and the estimated CIR.

The results obtained by varying the timing offset $\varepsilon_{t o}$, and the carrier offset $\varepsilon_{c o}$ are provided in Figure 2 parts (a) and (b) respectively. As can be seen in Figure 2 parts (a) there is a slight degradation in the resulting CIR estimate due to timing offset which is normally expected; however a-BLUE algorithm is insensitive to carrier phase offset. Both figures show the robustness of the a-BLUE algorithm to timing and carrier phase offsets.

\section{CONCLUSION}

This paper demonstrates the BLUE and a-BLUE CIR estimation algorithms for channels with long delay spreads, where the number of training symbols can be insufficient to support the length of the channel. In particular we show that a-BLUE initial channel estimation algorithm significantly outperforms the standard least squares and correlation based initial channel estimation algorithms achieving the same computational complexity. This feature makes the a-BLUE algorithm an attractive choice for receivers employing channel estimate based

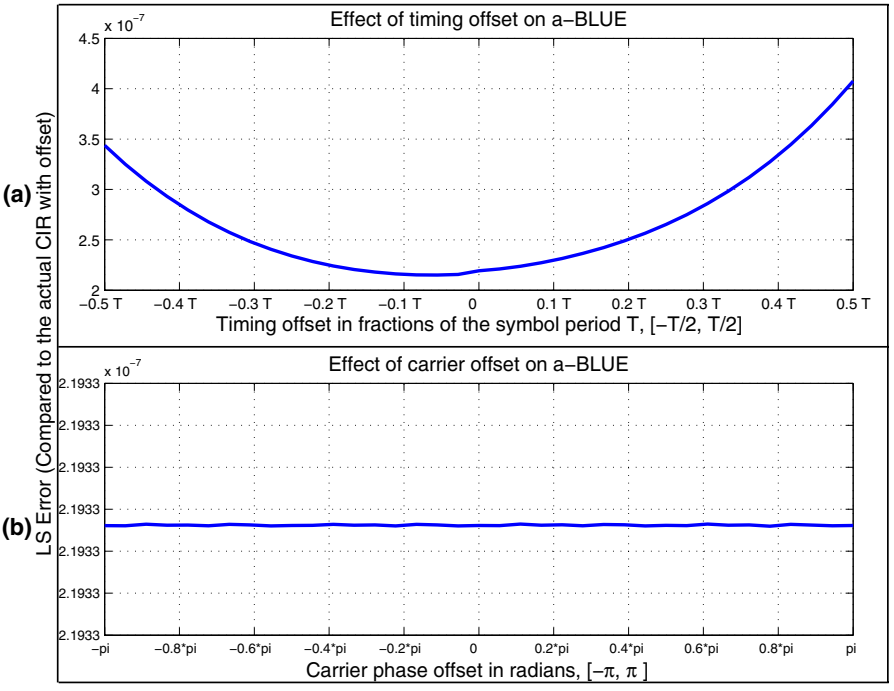

Fig. 2. Simulation results showing the robustness of the a-BLUE algorithm to (a) timing offset, and (b) carrier recovery phase offset.

(indirect) equalizers [5], or for receivers with direct adaptive equalizers where a quick and reliable channel information is needed for equalizer tap weight initialization.

We also demonstrated the robustness of the a-BLUE algorithm to timing and carrier offsets when there is no multipath present.

\section{ACKNOWLEDGMENTS}

This research was funded in part by Zenith Electronics Corporation.

\section{REFERENCES}

[1] H. Arslan, G. E. Bottomley, "Channel estimation in narrowband wireless communication systems," Wireless Comunications and Mobile Computing, vol. 1, pp. 201-219, 2001.

[2] ATSC Digital Television Standard, A/53, September 1995. (available from http://www.atsc.org/standards.html )

[3] E. de Carvalho, D. Slock, "Semi-blind methods for FIR multi-channel estimation," Chapter 7 in Signal Processing Advances in Wireless and Mobile Communications; Trends in Channel Estimation and Equalization, G. Giannakis, Y. Hua, P. Stoica, L. Tong, vol. 1, Prentice-Hall, 2001

[4] S. Özen, M. D. Zoltowski, M. Fimoff, "A Novel Channel Estimation Method: Blending Correlation and Least-Squares Based Approaches," Proceedings of ICASSP, v. 3, pp. 2281-2284, 2002.

[5] S. Özen, W. Hillery, M. D. Zoltowski, S. M. Nerayanuru, M. Fimoff, "Structured Channel Estimation Based Decision Feedback Equalizers for Sparse Multipath Channels with Applications to Digital TV Receivers," Proceedings of the Thirty-Sixth Asilomar Conference on Signals, Systems and Computers, vol. 1, pp. 558-564, November 3-6, 2002.

[6] S. Özen, M. Fimoff, C. Pladdy, S. M. Nerayanuru, M. D. Zoltowski, "Approximate Best Linear Unbiased Channel Estimation for Frequency Selective Multipath Channels with Long Delay Spreads," accepted to be published in the Proceedings of the Thirty-Seventh Asilomar Conference on Signals, Systems and Computers, November 2003.

[7] C. Pladdy, S. Özen, M. Fimoff, S. M. Nerayanuru, M. D. Zoltowski,"Best Linear Unbiased Channel Estimation for Frequency Selective Multipath Channels with Long Delay Spreads," accepted to be published in the Proceedings of Fall-2003 Vehicular Technology Conference, Orlando, Florida, USA, October-2003.

[8] G. A. F. Seber, Linear Regresion Analysis, John Wiley and Sons, 1977.

[9] H-K Song, "A channel estimation using sliding window aproach and tuning algorithm for MLSE," IEEE Communications Letters, vol. 3, pp. 211-213, 1999 